\title{
Association between the expression levels of IL-6 and IL-6R in the hepatocellular carcinoma microenvironment and postoperative recurrence
}

\author{
MAO ZHANG ${ }^{1 *}$, SHUN ZHANG $^{1 *}$, ZHENJIE YANG $^{1}$, JIE HU $^{2}$, WEIYU HU ${ }^{1}$, \\ PENG SUN ${ }^{1}$, LIQUN WU ${ }^{1}$ and BING HAN ${ }^{1}$
}

${ }^{1}$ Department of Hepatobiliary and Pancreatic Surgery, The Affiliated Hospital of Qingdao University, Qingdao, Shandong 266003; ${ }^{2}$ Department of General Surgery, The Third Xiangya Hospital of Central South University, Changsha, Hunan 410006, P.R. China

Received February 23, 2018; Accepted September 12, 2018

DOI: $10.3892 / \mathrm{ol} .2018 .9557$

\begin{abstract}
The tumor microenvironment of hepatocellular carcinoma (HCC) is a complex system, involving mutual promotion and inhibition between cells and molecules. It results in alterations to inflammatory-associated factors, including a decrease in interleukin (IL)-2 and an increase in tumor necrosis factor, together with a characteristic elevation of IL-6. Following the synthesis and release of IL-6, HCC is stimulated through IL-6 binding to the IL-6 receptor (IL-6R). In the present study, immunohistochemistry was performed to investigate the expression levels of IL-6 and IL-6R in 92 patients with $\mathrm{HCC}$, and the association between IL-6/IL-6R expression levels and tumor recurrence was examined. Notably increased expression levels of IL-6 and IL-6R were observed in the HCC microenvironment, and recurrence occurred earlier in patients with high IL-6/IL-6R expression levels compared with those with low expression levels $(\mathrm{P}<0.05)$. However, there was no significant difference in overall survival between patients in the two groups $(\mathrm{P}>0.05)$. Platelet levels $<100 \times 10^{9} / 1$, tumor-node-metastasis stage IIIa and high expression levels of IL-6/IL-6R were independent risk factors for postoperative recurrence $(\mathrm{P}<0.05)$. The present study proposed that high IL-6 and IL-6R expression in the HCC microenvironment promotes postoperative tumor recurrence, suggesting that
\end{abstract}

Correspondence to: Dr Bing Han or Professor Liqun Wu, Department of Hepatobiliary and Pancreatic Surgery, The Affiliated Hospital of Qingdao University, 16 Jiangsu Road, Qingdao, Shandong 266003, P.R. China

E-mail: 18661808308@163.com

E-mail: wulq581031@163.com

${ }^{*}$ Contributed equally

Key words: hepatocellular carcinoma microenvironment, inflammation factors interleukin-6, interlukin-6 receptor, immunohistochemistry, recurrence these may be potential predictors of recurrence, and may be used as possible therapeutic targets to enhance the long-term survival of patients.

\section{Introduction}

Hepatocellular carcinoma (HCC) is the sixth most common malignancy in the world, and its morbidity and mortality rates are ranked fourth and third, respectively, in China (1). Therefore, HCC is a serious public health concern (2). Progress has been made in the treatment of HCC, and the 5-year survival rate is now $\sim 70 \%$ following early liver resection or liver transplantation. However, $>80 \%$ of patients experience tumor recurrence following surgery, and relapse frequently reflects a lack of effective adjuvant therapy, making patient prognosis following surgical excision unsatisfactory $(3,4)$. Therefore, identifying novel indicators to prevent postoperative HCC recurrence and improve long-term survival is urgently required.

The occurrence and development of HCC are closely associated with the tumor microenvironment. Numerous reports have demonstrated the role of tumor-associated macrophages (TAMs) in promoting tumor blood vessel growth, lymphangiogenesis, immune suppression and other mechanisms in tumor progression (5,6). TAMs-M1 secrete interleukin (IL)-1 $\beta$, IL-6 and tumor necrosis factor- $\alpha$, and exert their antitumor effects by presenting tumor antigens to cytotoxic T lymphocytes $(7,8)$. TAMs-M2 inhibit the immune response of tumor cells via the action of cytokines, including IL-4, IL-13, IL-10 and prostaglandin E2 in the tumor microenvironment. Additionally, the release of IL-10, IL-6 and transforming growth factor may directly or indirectly promote tumor angiogenesis, growth and metastasis $(9,10)$.

The role of IL-6 in HCC is controversial. Yang et al (11) observed that lower levels of IL-6 expression in HCC prolonged overall survival (OS) and reduced the likelihood of tumor recurrence. However, Cho et al (12) reported that high levels of serum IL-6 in patients with HCC predicted long-term disease-free survival (DFS). Although IL-6 is closely associated with the occurrence, development and recurrence of HCC, these discrepancies indicate that the function of IL- 6 in HCC requires further 
study. Additionally, the majority of studies have investigated the association between HCC prognosis and serum IL-6 levels, as opposed to tissue IL-6 levels $(13,14)$. Following its synthesis and release, IL-6 binds to IL-6R on the surface of HCC cells (15). Therefore, in the present study, immunohistochemistry (IHC) was used to detect the levels of IL-6 and IL-6R in the tumor tissue of 92 patients with HCC. The association between IL-6 levels in tumor tissue and postoperative recurrence was analyzed. The current study may provide the basis for an evaluation of postoperative recurrence in patients with HCC.

\section{Materials and methods}

Patient information. Paraffin-embedded specimens that had been surgically removed from 92 patients with HCC, between January 2000 and December 2012 at the Department of Hepatobiliary and Pancreatic Surgery, The Affiliated Hospital of Qingdao University (Qingdao, China), were retrospectively collected. The patients comprised 75 males and 17 females (male: female, 4.41:1), aged 31-83 years (median age, 55.27 years). Inclusion criteria were: i) A postoperative pathological diagnosis of HCC; and ii) tumor-node-metastasis (TNM) staging TnNOM0. Exclusion criteria were: i) Anti-cancer treatment prior to surgery; ii) serious complications or mortality within 30 days post-surgery; iii) non-tumor associated mortality; and iv) incomplete clinical, pathological, or surgical data. According to the American Joint Committee on Cancer (7th edition) staging system (16), patients were divided into TNM stage $\mathrm{I}(\mathrm{n}=80)$, stage II $(n=5)$ and stage IIIa $(n=7)$. According to the World Health Organization classification of tumor histological grade, tumors were classed as well differentiated $(n=9)$, moderately differentiated $(n=61)$, or poorly differentiated $(n=22)$. The classification of liver function using Child-Pugh scoring grouped 89 cases into A grade, and three cases into B grade. The Ethics Committee of The Affiliated Hospital of Qingdao University approved the present study. Written informed consent was obtained from all patients.

IHC. Pathological specimens from $92 \mathrm{HCC}$ tissue samples were fixed in $4 \%$ neutral formaldehyde solution at room temperature for $24 \mathrm{~h}$, embedded in paraffin and sliced into 4-mm sections. Paraffin was removed with xylene (15 min; Shanghai Macklin Biochemical Co., Ltd, Shanghai, China) and the sections were rehydrated through a graded alcohol series (anhydrous ethanol I, $5 \mathrm{~min}$; anhydrous ethanol II, $5 \mathrm{~min}$; 95\% ethanol, $3 \mathrm{~min}$; $90 \%$ ethanol, $3 \mathrm{~min}$; $80 \%$ ethanol, $2 \mathrm{~min}$; and $70 \%$ ethanol, 2 min) (Shanghai Macklin Biochemical Co., Ltd, Shanghai, China). Following antigen retrieval with $10 \mathrm{mM}$ citrate buffer (pH 6; Beijing Jimei Biotechnology Co., Ltd., Beijing, China) at $100^{\circ} \mathrm{C}$ for $10-15 \mathrm{~min}$, endogenous peroxidase activity was blocked with $3 \%$ hydrogen peroxide for $10 \mathrm{~min}$ at $37^{\circ} \mathrm{C}$. Subsequently, the tissue sections were incubated with rabbit polyclonal antibodies against human IL-6 (1:500 dilution; cat. no. Ab9324; Abcam, Cambridge, MA, USA) or IL-6R (1:200 dilution; cat. no. Ab128008; Abcam), overnight at $4^{\circ} \mathrm{C}$ in a humidified chamber. The MaxVision kit (Fuzhou Maixin Biotechnology Development Co., Ltd., Fujian, China) was used to detect primary antibodies, and the color was developed using 3,3'-diaminobenzidine chromogen substrate for $10 \mathrm{~min}$ at room temperature, and counterstained with hematoxylin at room temperature for $1 \mathrm{~min}$. Tissue sections were dehydrated in graded ethanol, cleared and finally mounted.

IHC evaluation. Two pathologists, who were blinded to the clinical data, interpreted the results simultaneously. If the interpretations were different, the results were discussed with a third party until a consensus was reached. Under an optical microscope, positively stained cells displayed yellow or brown coloring in the cytoplasm. A total of ten fields were observed under high magnification (X400), and the percentage of positive cells was calculated. Cells without coloring scored 0 , while those displaying light yellow, brown and tan scored 1, 2 and 3 points, respectively. A second score was obtained by determining the percentage of positive cells: $<5 \%, \geq 5 \%, \geq 26 \%, \geq 51 \%$ and $\geq 75 \%$ scored $0,1,2,3$ and 4 points, respectively. The two scores were summed, and a total of $\leq 4$ represented low expression levels, while a score of $>4$ represented high expression levels. All patients were divided into high or low groups according to the results from the IHC. Patients with high expression levels of IL-6 and IL-6R were classified as the high expression group, while those with low expression levels of IL-6 and IL-6R, high IL-6 and low IL-6R, or low IL-6 and high IL-6R expression levels were designated as the low expression group. Representative figures are displayed in Fig. 1.

Patient follow-up. Close follow-up was performed for all cases that met the inclusion criteria. Follow-up took the form of outpatient visits, telephone calls, or correspondence. For the first 2 years following the review, follow-up took place every 3 months. For the subsequent 3 years, follow-up took place every 6 months; thereafter, an annual review took place. Reviews included an $\alpha$-fetoprotein liver function test, abdominal ultrasound and chest radiography. If necessary, enhanced CT or magnetic resonance imaging were performed together with biopsy and other tests. Recurrence was defined as the confirmation of new or intrahepatic lesions by imaging studies or biopsy. The DFS time was defined as the time from surgery until recurrence or the end of follow-up. The OS time was defined as the time from surgery until mortality or the end of follow-up. DFS and OS were calculated on a monthly basis, and the follow-up deadline was March 2017.

Statistical analysis. All statistical analyses were performed using SPSS software version 19.0 (IBM Corp., Armonk, NY, USA). The $\chi^{2}$ test, continuous correction $\chi^{2}$ test and Fisher's exact test were used to analyze the association between IL-6/IL-6R expression and clinicopathological characteristics. DFS and OS were assessed using Kaplan-Meier curves. The log-rank test was used to compare the expression of IL-6/IL-6R with recurrence and survival. The Cox proportional hazards model was used to screen variables for unilateral and multivariate analysis of $\mathrm{HCC}$ recurrence. A two-sided $\mathrm{P}<0.05$ was considered to indicate a statistically significant difference.

\section{Results}

Association between the expression levels of IL-6/IL-6R in a $H C C$ microenvironment and clinicopathological characteristics. The expression levels of IL-6 and IL-6R were detected by IHC. A proportion of interstitial cells were weakly stained 

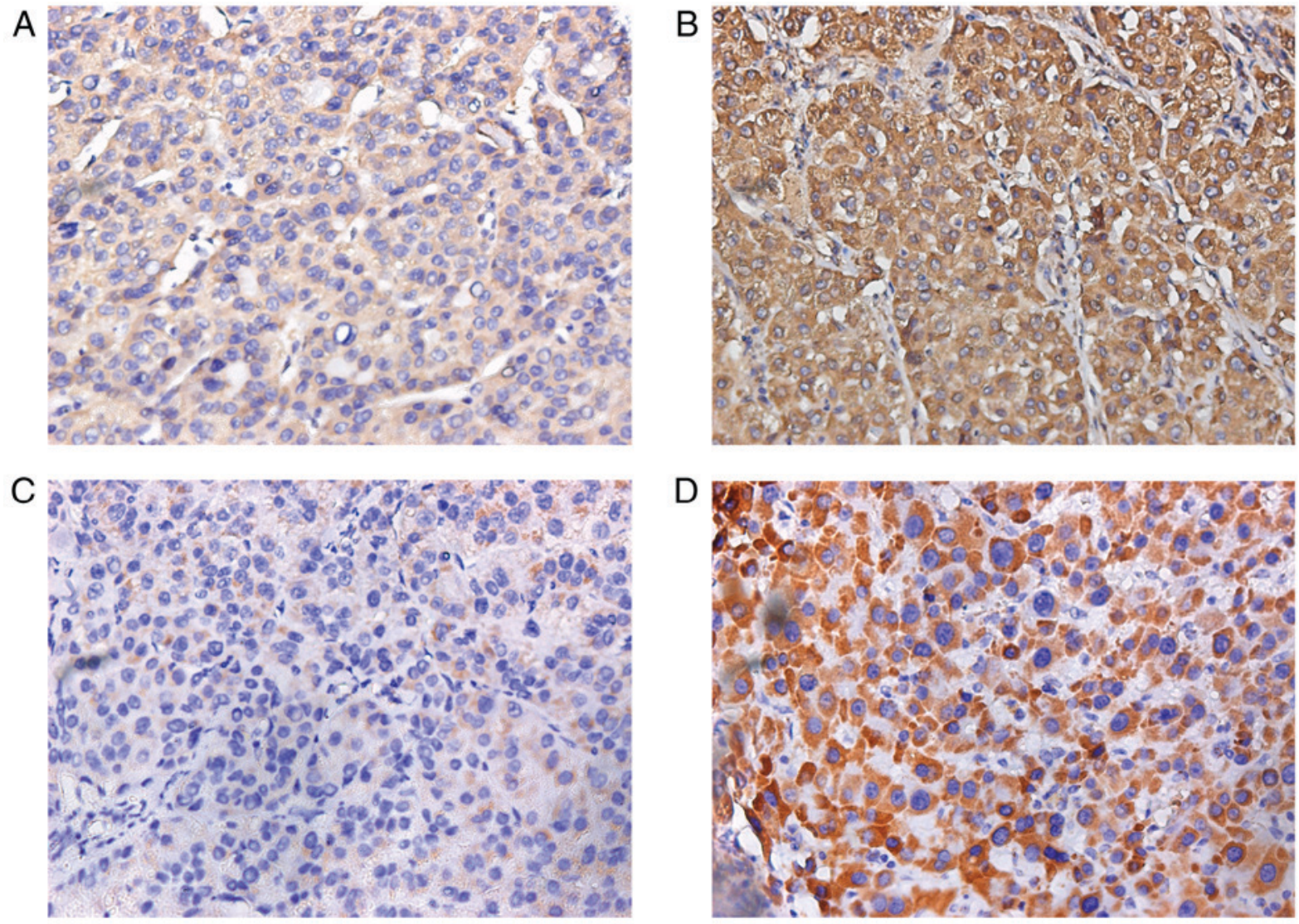

Figure 1. Expression levels of IL-6 and IL-6R as detected by immunohistochemistry. (A) Low expression and (B) high expression of IL-6 in the HCC microenvironment (magnification, $\mathrm{x} 400$ ). (C) Low expression and (D) high expression of IL-6R in the HCC microenvironment (magnification, $\mathrm{x} 400$ ). IL, interleukin; HCC, hepatocellular carcinoma.

with highly diffused or diffused brown granules (Fig. 1). High expression levels of IL-6 and IL-6R was detected in $22.8 \%$ (21/92) of HCC tissues. The associations between IL-6/IL-6R expression in the HCC microenvironment and various clinicopathological characteristics of patients with HCC were analyzed by the $\chi^{2}$ test, as displayed in Table I. No significant associations were observed between IL-6/IL-6R expression and sex, age or liver cirrhosis $(\mathrm{P}=0.128,0.606$ and 0.439 , respectively; Table I).

Association between the expression levels of $I L-6 / I L-6 R$ and postoperative prognosis. The average follow-up time was 44.05 \pm 24.27 months (range, 4-114.5 months), and the 1-, 3- and 5-year DFS rates were 48.91, 32.61 and $17.39 \%$, respectively. The overall median DFS time was 11.70 months [95\% confidence interval (CI): 4.27-19.13], while the median DFS in the low expression group was 22.20 months (95\% CI: 10.00-34.40) and 7.50 months (95\% CI: 5.82-9.18) in the high expression group. A significant difference in DFS was detected between the low expression and high expression groups $\left(\chi^{2}=6.855\right.$, $\mathrm{P}=0.009$; Fig. 2A).

OS rates at 1, 3 and 5 years were 89.13, 63.04 and $29.35 \%$, respectively. The median OS time was 65.00 months $(95 \% \mathrm{CI}$ : 46.09-83.91), while median OS was 67.30 months (95\% CI: $31.65-102.96)$ in the low expression group and 57.80 months (95\% CI: 26.65-88.95) in the high expression group. There was no significant difference between the two groups $\left(\chi^{2}=0.598\right.$, $\mathrm{P}=0.439$; Fig. 2B).
Univariate and multivariate analysis of DFS (Cox risk regression model). The factors that may influence HCC recurrence were individually included in the Cox proportional hazard model for univariate analysis. The results demonstrated that TNM stage, IL-6/IL-6R expression, and levels of platelet (PLT), prothrombin (PT), alanine aminotransferase (ALT) and aspartate aminotransferase (AST) significantly affected the postoperative recurrence of $\mathrm{HCC}(\mathrm{P}<0.05)$, while sex, age, and hepatitis $\mathrm{B}$ virus (HBV) infection had no significant effect $(\mathrm{P}>0.05)$. Those factors identified as significant in univariate analysis underwent multivariate Cox regression analysis, which demonstrated that the levels of PT, ALT and AST did not significantly affect postoperative recurrence in patients with HCC $(\mathrm{P}>0.05)$. However, PLT levels $<100 \times 10^{9} / 1$ $(\mathrm{P}=0.008)$, TNM stage IIIa $(\mathrm{P}=0.012)$ and high expression levels of IL-6/IL-6R $(\mathrm{P}=0.013)$ were independent risk factors for recurrence.

\section{Discussion}

HCC is a serious public health concern (2). Although a variety of treatments are currently available for HCC, surgical resection and liver transplantation remain the first choice (17), and relapse frequently reflects a lack of effective adjuvant therapy, making patient prognosis post-surgical excision unsatisfactory (4). Therefore, identifying an effective indicator to prevent the postoperative recurrence of $\mathrm{HCC}$ is crucial. Inflammation and immune-associated factors are known to have an impact 
Table I. Correlation between IL-6/IL-6R expression and clinicopathological characteristics.

\begin{tabular}{|c|c|c|c|c|c|}
\hline \multirow[b]{2}{*}{ Characteristics } & \multirow[b]{2}{*}{ Number } & \multicolumn{2}{|c|}{ IL-6 and IL-6R, n (\%) } & \multirow[b]{2}{*}{$\chi^{2}$} & \multirow[b]{2}{*}{ P-value } \\
\hline & & High & Low & & \\
\hline Sex & & & & 2.321 & $0.128^{\mathrm{b}}$ \\
\hline Male & 75 & $20(26.7)$ & $55(73.3)$ & & \\
\hline Female & 17 & $1(5.9)$ & $16(94.1)$ & & \\
\hline Age (y) & & & & 0.266 & $0.606^{\mathrm{a}}$ \\
\hline$<50$ & 26 & $5(19.2)$ & $21(80.8)$ & & \\
\hline$\geq 50$ & 66 & $16(24.2)$ & $50(75.8)$ & & \\
\hline Alcoholism & & & & 0.522 & $0.470^{\mathrm{a}}$ \\
\hline Yes & 25 & $7(28.0)$ & $18(72.0)$ & & \\
\hline No & 67 & $14(20.9)$ & $53(79.1)$ & & \\
\hline HBV infection & & & & 0.030 & $0.862^{\mathrm{b}}$ \\
\hline Yes & 82 & $18(22.0)$ & $64(78.0)$ & & \\
\hline No & 10 & $3(30.0)$ & $7(70.0)$ & & \\
\hline TBIL level $(\mu \mathrm{mol} / \mathrm{l})$ & & & & 0.315 & $0.575^{\mathrm{b}}$ \\
\hline$\leq 22$ & 80 & $17(21.3)$ & $63(78.7)$ & & \\
\hline$>22$ & 12 & $4(33.3)$ & $8(66.7)$ & & \\
\hline PT (s) & & & & - & $-1.000^{\mathrm{c}}$ \\
\hline$\leq 14$ & 90 & $21(23.3)$ & $69(76.7)$ & & \\
\hline$>14$ & 2 & $0(0.0)$ & $2(100.0)$ & & \\
\hline ALB level (g/l) & & & & 0.000 & $1.000^{\mathrm{b}}$ \\
\hline$<35$ & 11 & $3(27.3)$ & $8(72.7)$ & & \\
\hline$\geq 35$ & 81 & $18(22.2)$ & $63(77.8)$ & & \\
\hline ALT level (U/1) & & & & 0.010 & $0.920^{\mathrm{b}}$ \\
\hline$\leq 60$ & 73 & $16(21.9)$ & $57(78.1)$ & & \\
\hline$>60$ & 19 & $5(26.3)$ & $14(73.7)$ & & \\
\hline AST level (U/l) & & & & 0.208 & $0.648^{\mathrm{a}}$ \\
\hline$\leq 42$ & 65 & $14(21.5)$ & $51(78.5)$ & & \\
\hline$>42$ & 27 & $7(25.9)$ & $20(74.1)$ & & \\
\hline PLT level $\left(10^{9} / 1\right)$ & & & & 0.514 & $0.473^{\mathrm{a}}$ \\
\hline$<100$ & 23 & $4(17.4)$ & $19(82.6)$ & & \\
\hline$\geq 100$ & 69 & $17(24.6)$ & $52(75.4)$ & & \\
\hline Liver cirrhosis & & & & 0.599 & $0.439^{\mathrm{b}}$ \\
\hline Yes & 11 & $1(9.0)$ & $10(91)$ & & \\
\hline No & 81 & $20(24.7)$ & $61(75.3)$ & & \\
\hline AFP level (ng/l) & & & & 0.027 & $0.870^{\mathrm{a}}$ \\
\hline$\leq 400$ & 67 & $15(22.4)$ & $52(77.6)$ & & \\
\hline$>400$ & 25 & $6(24.0)$ & $19(76.0)$ & & \\
\hline Child-Pugh grade & & & & - & $-1.000^{c}$ \\
\hline A & 89 & $21(23.6)$ & $68(76.4)$ & & \\
\hline B & 3 & $0(0.0)$ & $3(100)$ & & \\
\hline Tumor number & & & & - & $-1.000^{\mathrm{c}}$ \\
\hline Single & 88 & $29(33.0)$ & $59(67.0)$ & & \\
\hline Multiple & 4 & $1(25.0)$ & $3(75.0)$ & & \\
\hline Tumor size $(\mathrm{cm})$ & & & & 0.353 & $0.552^{\mathrm{b}}$ \\
\hline$\leq 5$ & 84 & $26(31.0)$ & $58(69.0)$ & & \\
\hline$>5$ & 8 & $4(50.0)$ & $4(50.0)$ & & \\
\hline Tumor margin $(\mathrm{cm})$ & & & & 0.390 & $0.532^{\mathrm{b}}$ \\
\hline$\leq 2$ & 82 & $20(24.4)$ & $62(75.6)$ & & \\
\hline$>2$ & 10 & $1(10.0)$ & $9(90.0)$ & & \\
\hline
\end{tabular}


Table I. Continued.

\begin{tabular}{|c|c|c|c|c|c|}
\hline \multirow[b]{2}{*}{ Characteristics } & \multirow[b]{2}{*}{ Number } & \multicolumn{2}{|c|}{ IL-6 and IL-6R, n (\%) } & \multirow[b]{2}{*}{$\chi^{2}$} & \multirow[b]{2}{*}{ P-value } \\
\hline & & High & Low & & \\
\hline \multicolumn{4}{|c|}{ Pathological differentiation } & 1.367 & $0.242^{\mathrm{b}}$ \\
\hline High & 8 & $0(0.0)$ & $8(100.0)$ & & \\
\hline Middle and low & 84 & $21(25.0)$ & $63(75.0)$ & & \\
\hline \multicolumn{4}{|c|}{ Microvascular tumor thrombus } & 0.309 & $0.578^{\mathrm{b}}$ \\
\hline Yes & 16 & $5(31.3)$ & $11(68.7)$ & & \\
\hline No & 76 & $16(21.1)$ & $60(78.9)$ & & \\
\hline \multicolumn{4}{|l|}{ Capsule invasion } & 0.083 & $0.774^{\mathrm{b}}$ \\
\hline Yes & 84 & $29(34.5)$ & 55 (65.5) & & \\
\hline No & 8 & $1(12.5)$ & $7(87.5)$ & & \\
\hline \multicolumn{4}{|l|}{ TNM stage } & 0.724 & $0.398^{\mathrm{b}}$ \\
\hline $\mathrm{I}+\mathrm{II}$ & 85 & $18(21.2)$ & 67 (78.8) & & \\
\hline IIIa & 7 & $4(57.1)$ & $3(42.9)$ & - & - \\
\hline
\end{tabular}

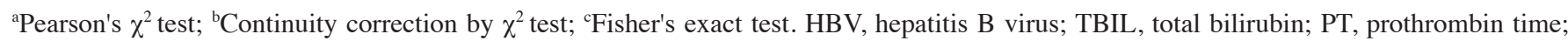
ALB, albumin; ALT, alanine aminotransferase; AST, aspartate aminotransferase; PLT, platelet; AFP, $\alpha$-fetoprotein; IL, interleukin; TNM, tumor, node, metastasis.
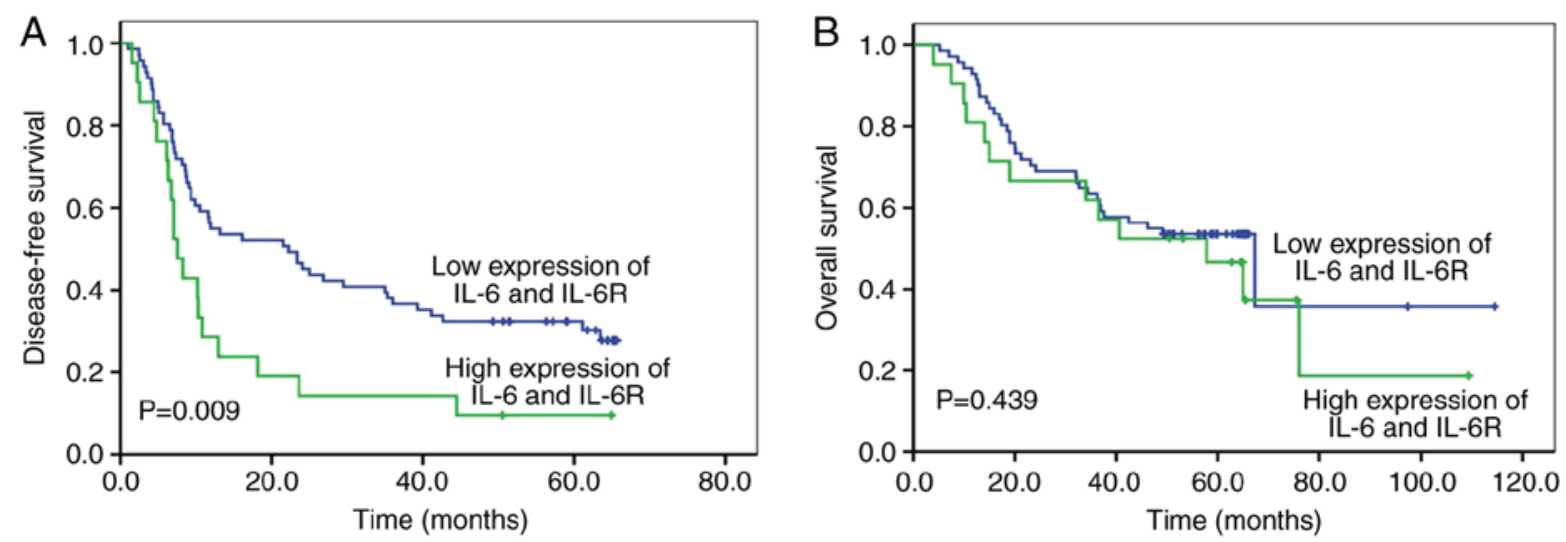

Figure 2. Kaplan-Meier curves presenting the association between IL-6/IL-6R expression levels and postoperative prognosis. (A) Disease-free survival rates of patients with high and low expression levels of IL-6/IL-6R in the HCC microenvironment. (B) Overall survival rates of patients with high and low expression levels of IL-6/IL-6R in the HCC microenvironment. IL, interleukin; HCC, hepatocellular carcinoma.

on long-term survival following liver resection in patients with HCC (18). Various studies have reported the association of IL-2, IL-15, IL-8, IL-6, IL-10 and other cytokines with the occurrence and development of HCC (19-22). In the present study, IL-6 expression levels as an indicator of HCC recurrence following surgery was evaluated. IL- 6 binds to IL-6R on the target-cell surface in order to activate the Janus kinase/signal transducers and activators of transcription (STAT) pathway, in the promotion of HCC (23-27). IHC was used to detect IL-6 and IL-6R expression levels in the tumor microenvironment of patients with HCC, with the aim of evaluating the long-term prognosis of HCC following surgical treatment.

Previous studies $(13,14,28)$ reported a strong association between high circulating blood levels of IL- 6 and poor prognosis in $\mathrm{HCC}$, and also reported that the higher the serum concentration of IL-6, the larger the HCC tumor volume, the more advanced the stage and the worse the prognosis. Yang et al (11) inhibited the activity of the IL-6-IL-3 signaling pathway through the overexpression of microRNA-26a, which reduced the effect of IL-6 in HCC, resulting in increased OS times, delayed relapse times and an improved prognosis; together, these results suggested that high levels of IL-6 may be positively correlated with poor prognosis. The present study demonstrated that high expression levels of IL-6 and IL-6R in the HCC microenvironment may be associated with markedly earlier tumor recurrence compared with low expression levels of IL-6 and IL-6R. This is in accordance with previous studies, which reported that high IL-6 and IL-6R expression levels in the HCC microenvironment may promote postoperative HCC recurrence. However, the present study also demonstrated that the OS times of patients with high IL-6 and IL-6R expression levels did not differ significantly compared with those 
Table II. Univariate and multivariate analysis of variables with disease-free survival.

\begin{tabular}{|c|c|c|c|c|}
\hline \multirow[b]{2}{*}{ Variables } & \multicolumn{2}{|c|}{ Univariate } & \multicolumn{2}{|c|}{ Multivariate } \\
\hline & $\mathrm{HR}(95 \% \mathrm{CI})$ & P-value & HR $(95 \%$ CI $)$ & P-value \\
\hline Sex (female vs. male) & $0.686(0.359-1.30)$ & 0.253 & - & - \\
\hline Age $(<50$ vs. $\geq 50)$ & $1.193(0.709-2.009)$ & 0.506 & - & - \\
\hline Alcoholism & $1.186(0.704-2.000)$ & 0.521 & - & - \\
\hline HBV infection & $1.196(0.546-2.619)$ & 0.655 & - & - \\
\hline TBIL level ( $\leq 22$ vs. $>22)$ & $1.713(0.897-3.270)$ & 0.103 & - & - \\
\hline PT level ( $\leq 14$ vs. $>14)$ & $7.718(1.801-33.074)$ & $0.006^{\mathrm{a}}$ & - & - \\
\hline ALB level $(<35$ vs. $\geq 35)$ & $0.714(0.326-1.562)$ & 0.399 & - & - \\
\hline ALT level $(\leq 60$ vs. $>60)$ & $1.855(1.055-3.261)$ & $0.032^{\mathrm{a}}$ & - & - \\
\hline AST level ( $\leq 42$ vs. $>42)$ & $2.145(1.289-3.568)$ & $0.003^{\mathrm{a}}$ & - & - \\
\hline PLT level (<100 vs. $\geq 100)$ & $1.875(1.110-3.166)$ & $0.019^{\mathrm{a}}$ & $2.267(1.237-4.155)$ & $0.008^{\mathrm{a}}$ \\
\hline Liver cirrhosis & $1.228(0.609-2.476)$ & 0.567 & - & - \\
\hline AFP level ( $\leq 400$ vs. $>400)$ & $1.495(0.888-2.517)$ & 0.131 & - & - \\
\hline Child-Pugh grade (A vs. B) & $1.292(0.316-5.284)$ & 0.722 & - & - \\
\hline Tumor number (single vs. multiple) & $2.666(0.962-7.383)$ & 0.059 & - & - \\
\hline Tumor size ( $\leq 5$ vs. $>5$ ) & $1.924(0.914-4.051)$ & 0.085 & - & - \\
\hline Tumor margin ( $\leq 2$ vs. $>2)$ & $0.445(0.178-1.110)$ & 0.082 & - & - \\
\hline Differentiation (high vs. middle and low) & $1.216(0.526-2.812)$ & 0.648 & - & - \\
\hline Microvascular tumor thrombus & $1.720(0.955-3.100)$ & 0.071 & - & - \\
\hline Capsule invasion & $1.246(0.500-3.104)$ & 0.637 & - & - \\
\hline TNM stage (I+II vs. IIIa) & $4.154(1.843-9.365)$ & $0.001^{\mathrm{a}}$ & $3.604(1.318-9.861)$ & $0.013^{\mathrm{a}}$ \\
\hline IL-6 and IL-6R (low vs. high) & $2.014(1.179-3.441)$ & $0.010^{\mathrm{a}}$ & $2.097(1.180-3.726)$ & $0.012^{\mathrm{a}}$ \\
\hline
\end{tabular}

${ }^{\mathrm{a}} \mathrm{P}<0.05$. HR, hazard ratio; CI, confidence interval; HBV, hepatitis B virus; TBIL, total bilirubin; PT, prothrombin time; ALB, albumin; ALT, alanine aminotransferase; AST, aspartate aminotransferase; PLT, platelet; AFP, $\alpha$-fetoprotein; IL, interlukin; TNM, tumor, node, metastasis.

with low expression levels, which contrasts with the results of Jang et al (13). This difference may be the result of recurrence patterns, treatment modalities following recurrence, baseline liver function and tumor number, which may influence OS following recurrence in patients with HCC (29-31).

The present study demonstrated that high IL-6 and IL-6R expression levels are an independent risk factor for the postoperative recurrence of HCC. Sheng et al (32) reported that preoperative IL-6 serum levels may be a potential biomarker for early prediction of hepatitis B virus-associated HCC recurrence. Additionally, Strassmann et al (33) demonstrated that IL-6 may regulate the state of cancer cachexia, causing the progression and worsening of disease, while He et al (34) reported that high IL-6 expression levels in HCC may be closely associated with the malignant progression of HCC. Together, these results indicate that IL-6 and IL-6R may serve an important role in the occurrence and development of cancer in the HCC microenvironment.

The present study also demonstrated that TNM stage IIIa may be an independent risk factor for the postoperative recurrence of $\mathrm{HCC}$, which is similar to the findings of Kim et al (35). In recent years, a variety of staging systems and clinicopathological factors have been combined in order to predict the prognosis of HCC (36), and Metwaly et al (37) reported that serum IL-6 levels positively correlated with HCC staging. This highlights the importance of early detection, prompt diagnosis and timely surgery in order to reduce the clinical staging of preoperative tumors, prevent postoperative recurrence and improve prognosis. The present study reports that there is no association between IL- 6 levels in the microenvironment and TNM staging, which may reflect the small number of cases analyzed.

Alterations in PLT levels are currently attributed to the effects of serum IL-6 levels. Elevated serum IL-6 promotes the expression of thrombopoietin, and cooperates with IL-3, IL-11 and stem cell factor to stimulate large-scale expansion and premature maturation of the bone marrow megakaryocyte cell line, which is involved in the formation of platelets (38). While serum IL-6 levels were not analyzed in the present study, no association between IL- 6 levels in the local microenvironment and PLT levels in the blood were reported. Therefore, levels of IL-6 in the local microenvironment are not thought to affect whole serum IL-6 expression levels. Moreover, PLT levels of $<100 \times 10^{9} / 1$ were demonstrated as an independent risk factor for postoperative HCC recurrence ( $R R=2.267$, Table II), which is consistent with the findings of Amano et al (39) and Kaneko et al (40).

In conclusion, the present study demonstrated that the expression levels of IL-6 and IL-6R in the HCC microenvironment may correlate with postoperative $\mathrm{HCC}$ recurrence. However, 
while the high expression levels of IL-6 and IL-6R promoted $\mathrm{HCC}$ recurrence, it did not affect postoperative survival time. Meanwhile, TNM stage IIIa, PLT $<100 \times 10^{9} / 1$ and high expression levels of IL-6/IL-6R were independent risk factors for postoperative recurrence. This preliminary study of IL-6 and IL-6R expression levels in the HCC tumor microenvironment is a basis for future large-scale studies into disease prognosis. Further work may also allow the identification of novel treatments for HCC. Although antibodies against IL- 6 have been marketed as drugs for the treatment of rheumatoid arthritis, no studies have reported the use of anti-IL-6 antibodies in HCC treatment. However, they may be a promising and effective means of enhancing long-term survival in patients with HCC.

\section{Acknowledgements}

The authors wish to thank Dr Sarah Williams for editing the English text in the draft of this manuscript.

\section{Funding}

The study was supported by grants from the Key Research and Development Plan of Shandong Province (grant no. 2018GSF118233), the Science and Technology Plan of Qingdao City Shinan District (grant no. 2018-4-018-YY), and the Natural Science Foundation of Shandong Province (grant no. ZR2012HQ039).

\section{Availability of data and materials}

The datasets used and/or analyzed during the current study are available from the corresponding author on reasonable request.

\section{Authors' contributions}

$\mathrm{BH}, \mathrm{LW}, \mathrm{SZ}$ and $\mathrm{WH}$ contributed to the study design. MZ, $\mathrm{ZY}, \mathrm{JH}$ and PS contributed to data analysis. ZY, JH and PS contributed to the collection of tissue samples and patient data. MZ and SZ wrote the manuscript. All authors have read and approved the final version of the manuscript.

\section{Ethics approval and consent to participate}

The Ethics Committee of The Affiliated Hospital of Qingdao University approved the study. Written informed consent was obtained from all patients.

\section{Patient consent for publication}

Not applicable.

\section{Competing interests}

The authors declare that they have no competing interests.

\section{References}

1. Chen W, Zheng R, Baade PD, Zhang S, Zeng H, Bray F, Jemal A, Yu XQ and He J: Cancer Statistics in China, 2015. CA Cancer J Clin 66: 115-132, 2016.
2. Chen C and Wang G: Mechanisms of hepatocellular carcinoma and challenges and opportunities for molecular targeted therapy. World J Hepatol 7: 1964-1970, 2015.

3. Llovet JM, Schwartz M and Mazzaferro V: Resection and liver transplantation for hepatocellular carcinoma. Semin Liver Dis 25: 181-200, 2005.

4. Poon RT: Prevention of recurrence after resection of hepatocellular carcinoma: A daunting challenge. Hepatology 54: 757-759, 2011.

5. Coffelt SB, Hughes R and Lewis CE: Tumor-associated macrophages: Effectors of angiogenesis and tumor progression. Biochim Biophys Acta 1796: 11-18, 2009.

6. Quatromoni JG and Eruslanov E: Tumor-associated macrophages: Function, phenotype, and link to prognosis in human lung cancer. Am J Transl Res 4: 376-389, 2012.

7. Esser N, Legrand-Poels S, Piette J, Scheen AJ and Paquot N: Inflammation as a link between obesity, metabolic syndrome and type 2 diabetes. Diabetes Res Clin Pract 105: 141-150, 2014.

8. Wang YC, He F, Feng F, Liu XW, Dong GY, Qin HY, Hu XB Zheng MH, Liang L, Feng L, et al: Notch signaling determines the M1 versus M2 polarization of macrophages in antitumor immune responses. Cancer Res 70: 4840-4849, 2010.

9. Mantovani A, Sica A, Sozzani S, Allavena P, Vecchi A and Locati M: The chemokine system in diverse forms of macrophage activation and polarization. Trends Immunol 25: 677-686, 2004.

10. Wang HW and Joyce JA: Alternative activation of tumor-associated macrophages by IL-4: Priming for protumoral functions. Cell Cycle 9: 4824-4835, 2010

11. Yang X, Liang L, Zhang XF, Jia HL, Qin Y, Zhu XC, Gao XM, Qiao P, Zheng Y, Sheng YY, et al: MicroRNA-26a suppresses tumor growth and metastasis of human hepatocellular carcinoma by targeting interleukin-6-Stat3 pathway. Hepatology 58: 158-170, 2013.

12. Cho HJ, Kim SS, Ahn SJ, Park SY, Park JH, Kim JK, Wang HJ, Cheong JY and Cho SW: Low serum interleukin-6 levels as a predictive marker of recurrence in patients with hepatitis B virus related hepatocellular carcinoma who underwent curative treatment. Cytokine 73: 245-252, 2015.

13. Jang JW, Oh BS, Kwon JH, You CR, Chung KW, Kay CS and Jung HS: Serum interleukin-6 and C-reactive protein as a prognostic indicator in hepatocellular carcinoma. Cytokine 60: 686-693, 2012.

14. Ohishi W, Cologne JB, Fujiwara S, Suzuki G, Hayashi T, Niwa Y, Akahoshi M, Ueda K, Tsuge M and Chayama K: Serum interleukin-6 associated with hepatocellular carcinoma risk: A nested case-control study. Int J Cancer 134: 154-163, 2014.

15. Ataie-Kachoie P, Pourgholami MH and Morris DL: Inhibition of the IL-6 signaling pathway: A strategy to combat chronic inflammatory diseases and cancer. Cytokine Growth Factor Rev 24: 163-173, 2013.

16. Edge SB and Compton CC: The American Joint Committee on Cancer: The 7th Edition of the AJCC Cancer Staging Manual and the Future of TNM. Ann Surg Oncol 17: 1471-1474, 2010.

17. Carr BI: Hepatocellular carcinoma: Current management and future trends. Gastroenterology 127 (5 Suppl 1): S218-S124, 2004.

18. Chen L, Zhang Q, Chang W, Du Y, Zhang H and Cao G: Viral and host inflammation-related factors that can predict the prognosis of hepatocellular carcinoma. Eur J Cancer 48: 1977-1987, 2012.

19. Hsia CY, Huo TI, Chiang SY, Lu MF, Sun CL, Wu JC, Lee PC, Chi CW, Lui WY and Lee SD: Evaluation of interleukin-6, interleukin-10 and human hepatocyte growth factor as tumor markers for hepatocellular carcinoma. Eur J Surg Oncol 33: 208-212, 2007.

20. Mosser DM and Zhang X: Interleukin-10: New perspectives on an old cytokine. Immunol Rev 226: 205-218, 2008.

21. Ren Y, Poon RT, Tsui HT, Chen WH, Li Z, Lau C, Yu WC and Fan ST: Interleukin-8 serum levels in patients with hepatocellular carcinoma: Correlations with clinicopathological features and prognosis. Clin Cancer Res 9: 5996-6001, 2003.

22. Zhou H, Huang H, Shi J, Zhao Y, Dong Q, Jia H, Liu Y, Ye Q, Sun H, Zhu X, et al: Prognostic value of interleukin 2 and interleukin 15 in peritumoral hepatic tissues for patients with hepatitis B-related hepatocellular carcinoma after curative resection. Gut 59: 1699-1708, 2010.

23. Hunter CA and Jones SA: IL-6 as a keystone cytokine in health and disease. Nat Immunol 16: 448-457, 2015.

24. Ataie-Kachoie P, Pourgholami MH, Richardson DR and Morris DL: Gene of the month: Interleukin 6 (IL-6). J Clin Pathol 67: 932-937, 2014. 
25. Bollrath J, Phesse TJ, von Burstin VA, Putoczki T, Bennecke M, Bateman T, Nebelsiek T, Lundgren-May T, Canli O, Schwitalla S, et al: gp130-mediated Stat3 activation in enterocytes regulates cell survival and cell-cycle progression during colitis-associated tumorigenesis. Cancer cell 15: 91-102, 2009.

26. Kujawski M, Kortylewski M, Lee H, Herrmann A, Kay H and Yu H: Stat 3 mediates myeloid cell-dependent tumor angiogenesis in mice. J Clin Invest 118: 3367-3377, 2008.

27. Wang XH, Liu BR, Qu B, Xing H, Gao SL, Yin JM, Wang XF and Cheng YQ: Silencing STAT3 may inhibit cell growth through regulating signaling pathway, telomerase, cell cycle, apoptosis and angiogenesis in hepatocellular carcinoma: Potential uses for gene therapy. Neoplasma 58: 158-171, 2011.

28. Soresi M, Giannitrapani L, D'Antona F, Florena AM, La Spada E, Terranova A, Cervello M, D'Alessandro $\mathrm{N}$ and Montalto G: Interleukin-6 and its soluble receptor in patients with liver cirrhosis and hepatocellular carcinoma. World J Gastroenterol 12: 2563-2568, 2006.

29. Hanazaki K, Kajikawa S, Shimozawa N, Mihara M, Shimada K, Hiraguri M, Koide N, Adachi W and Amano J: Survival and recurrence after hepatic resection of 386 consecutive patients with hepatocellular carcinoma. J Am Coll Surg 191: 381-388, 2000.

30. Poon RT, Fan ST, Lo CM, Liu CL and Wong J: Long-term survival and pattern of recurrence after resection of small hepatocellular carcinoma in patients with preserved liver function: Implications for a strategy of salvage transplantation. Ann Surg 235: 373-382, 2002.

31. Shiina S, Tateishi R, Arano T, Uchino K, Enooku K, Nakagawa H, Asaoka Y, Sato T, Masuzaki R, Kondo Y, et al: Radiofrequency ablation for hepatocellular carcinoma: 10-year outcome and prognostic factors. Am J Gastroenterol 107: 569-577, 2012.

32. Sheng T, Wang B, Wang SY, Deng B, Qu L, Qi XS, Wang XL, Deng GL and Sun X: The relationship between serum interleukin-6 and the recurrence of hepatitis B virus related hepatocellular carcinoma after curative resection. Medicine (Baltimore) 94: e941, 2015.
33. Strassmann G, Fong M, Kenney JS and Jacob CO: Evidence for the involvement of interleukin 6 in experimental cancer cachexia. J Clin Invest 89: 1681-1684, 1992.

34. He G, Dhar D, Nakagawa H, Font-Burgada J, Ogata H, Jiang Y, Shalapour S, Seki E, Yost SE, Jepsen K, et al: Identification of liver cancer progenitors whose malignant progression depends on autocrine IL-6 Signaling. Cell 155: 384-396, 2013.

35. Kim KH and Choi YK: Long-term survival after resection of hepatocellular carcinoma. Korean J Hepatobiliary Pancreat Surg 16: 98-104, 2012.

36. Chen TW, Chu CM, Yu JC, Chen CJ, Chan DC, Liu YC and Hsieh CB: Comparison of clinical staging systems in predicting survival of hepatocellular carcinoma patients receiving major or minor hepatectomy. Eur J Surg Oncol 33: 480-487, 2007.

37. Metwaly HA, Al-Gayyar MM, Eletreby S, Ebrahim MA and El-Shishtawy MM: Relevance of serum levels of interleukin-6 and Syndecan-1 in patients with hepatocellular carcinoma. Sci Pharm 80: 179-188, 2012

38. Erickson-Miller CL, Chadderton A, Gibbard A, Kirchner J, Pillarisetti K, Baker K, Pandite L, El-Hariry I, Mostafa Kamel Y, Liu Y, et al: Thrombopoietin receptor levels in tumor cell lines and primary tumors. J Oncol 2010: 135354, 2010.

39. Amano $\mathrm{H}$, Tashiro $\mathrm{H}$, Oshita A, Kobayashi T, Tanimoto $\mathrm{Y}$, Kuroda S, Tazawa H, Itamoto T, Asahara $\mathrm{T}$ and Ohdan $\mathrm{H}$ : Significance of platelet count in the outcomes of hepatectomized patients with hepatocellular carcinoma exceeding the milan criteria. J Gastrointest Surg 15: 1173-1181, 2011.

40. Kaneko K, Shirai Y, Wakai T, Yokoyama N, Akazawa K and Hatakeyama K: Low preoperative platelet counts predict a high mortality after partial hepatectomy in patients with hepatocellular carcinoma. World J Gastroenterol 11: 5888-5892, 2005.

This work is licensed under a Creative Commons Attribution-NonCommercial-NoDerivatives 4.0 International (CC BY-NC-ND 4.0) License. 